ERRATUM

Journal of Artificial Organs Editorial Committee

\title{
Journal of Artificial Organs 2007: the year in review
}

\section{J Artif Organs (2008) 11:4-11 DOI 10.1007/s10047-008-0404-9}

Incomplete data were supplied for reference 22 . The full listing should be:

Nishinaka T, Tatsumi E, Katagiri N, Ohnishi H, Mizuno T, Shioya K, Tsukiya T, Homma A, Kashiwabara S, Tanaka H, Sato M, Taenaka Y. Up to 151 days of continuous animal perfusion with trivial heparin infusion by the application of a long-term durable antithrombogenic coating to a combination of a seal-less centrifugal pump and a diffusion membrane oxygenator. J Artif Organs 2007;10:240-244.

The publisher sincerely apologizes for any inconvenience this may have caused.

\section{Y. Sawa $(\square)$}

Division of Cardiovascular Surgery, Department of Surgery, Osaka University Graduate School of Medicine, 2-2 Yamadaoka, Suita,

Osaka 565-0871, Japan

Tel. +81-6-6979-3154; Fax +81-3-3879-3159

e-mail: sawa@surg1.medo.osaka-u.ac.jp

\section{E. Tatsumi}

Department of Artificial Organs, Advanced Medical Engineering Center, National Cardiovascular Center Research Institute, Suita, Japan

A. Funakubo

Department of Electronic and Computer Engineering, School of Science and Engineering, Tokyo Denki University, Tokyo, Japan

T. Horiuchi

Department of Chemistry for Materials, Faculty of Engineering, Mie University, Tsu, Japan

K. Iwasaki

Waseda Institute for Advanced Study, Waseda University, Tokyo, Japan

A. Kishida

Institute of Biomaterials and Bioengineering, Tokyo Medical and

Dental University, Tokyo, Japan
T. Masuzawa

Department of Mechanical Engineering, Ibaraki University, Hitachi, Japan

K. Matsuda

Emergency and Critical Care Medicine, University of Yamanashi

Hospital, Chuo, Japan

M. Nishimura

Division of Organ Regeneration Surgery, Tottori University Faculty of Medicine, Yonago, Japan

T. Nishimura

Cardiovascular Surgery, The University of Tokyo Hospital, Tokyo, Japan

Y. Tomizawa

Department of Cardiovascular Surgery, Tokyo Women's Medical University, Tokyo, Japan

T. Yamaoka

Department of Biomedical Engineering, Research Institute, National Cardiovascular Center, Suita, Japan

H. Watanabe

Division of Thoracic and Cardiovascular Surgery, Niigata University Graduate School of Medical and Dental Sciences, Niigata, Japan 